\title{
Cannabinoids for the treatment of pain: An update on recent clinical trials
}

\author{
Mark Ware MBBS MRCP MSc ${ }^{1}$, Pierre Beaulieu MD PhD²
}

\begin{abstract}
M Ware, P Beaulieu. Cannabinoids for the treatment of pain: An update on recent clinical trials. Pain Res Manage 2005;10(Suppl A):27A-30A.

Over the past five years, there has been a considerable increase in clinical research on cannabinoid use for a range of pain syndromes. Cannabinoid products are becoming available for research and clinical use, and pharmaceutical industry interest in the potential for cannabinoids in therapeutics is also gaining momentum. The present article summarizes recent clinical trial data in the field of pain management and suggests that the potential for cannabinoid therapy for chronic pain states is encouraging. Clinicians working in pain management should be aware of the options becoming available from the cannabinoid class of medications.
\end{abstract}

Key Words: Cannbinoid; Cannabis; Clinical trial; Pain; Review
The use of cannabinoids for the treatment of acute and chronic pain has a long and well-documented history. As reviewed elsewhere in this supplement, the mechanisms of cannabinoid action, which have recently been identified, provide evidence for a pain pathway mediated by cannabinoids (1). It is now recognized that $10 \%$ to $15 \%$ of patients with chronic pain use herbal cannabis as part of their treatment (2) and that this may result in reductions in opioid requirements (3-5). The use of cannabis or cannabinoids in the treatment of acute pain has not been as widely reported. Recent advances in cannabinoid pharmacology have resulted in increasing attention on the therapeutic potential of cannabinoids, and a number of preparations have been or are being developed and investigated in randomized controlled trials. The difficulties with conducting clinical trials on pain include the fact that pain is a subjective experience, and pain patients comprise a heterogenous group consisting of many different syndromes with a variety of physical, psychological and social problems. In addition to this, cannabinoids are associated with considerable social stigma, and cannabis for medical purposes has become a major politicolegal issue for many Western governments.

Historically, clinical data for the efficacy of cannabinoids in pain relief have been equivocal; a qualitative systematic review published in 2001 (6) concluded "cannabinoids are no more effective than codeine in controlling pain and have depressant effects on the central nervous system that limit their use. Their widespread introduction into clinical practice for pain management is therefore undesirable". The study was criticized for

\section{Les cannabinoïdes pour le traitement de la douleur : Le point sur les essais cliniques récents}

Au cours des cinq dernières années, on a effectué beaucoup de recherches cliniques sur l'utilisation des cannabinoïdes dans divers syndromes douloureux. Les dérivés des cannabinoïdes deviennent plus accessibles pour la recherche, leur utilisation clinique se répand et l'industrie pharmaceutique manifeste un intérêt croissant pour leur potentiel thérapeutique. Le présent article fait le point sur les essais cliniques récents dans le domaine du traitement de la douleur et confirme le potentiel des cannabinoïdes dans le traitement de la douleur chronique. Les médecins qui travaillent dans le domaine du contrôle de la douleur doivent être au courant des options qu'offrent les cannabinoïdes en tant que classe pharmacologique.

reaching conclusions that were not supported by the existing literature (7). Reports of the use of cannabis for pain continue to suggest a role for cannabinoids in pain management; nabilone has been used with reported benefit for chronic pain in clinical practice (8), and case reports (9) and case series (10) suggest that cannabinoids deserve further enquiry. In the past two years, a number of clinical trials have been reported investigating the efficacy of number of therapeutic cannabinoid compounds. The purpose of the present paper is to summarize these recent reports, to consolidate our current understanding of the effects of cannabinoids in pain management and to provide a stimulus for further research efforts.

\section{METHODS}

A review of publications relating to the use of cannabinoids in the treatment of pain in humans was conducted. The search was based on the authors' libraries of material and communications with other investigators. In addition, MEDLINE was searched for clinical trials of cannabinoids for acute and chronic pain; these were summarized and categorized according to the compound used, including naturally occurring and synthetic cannabinoids.

\section{Acute pain}

\section{RESULTS}

Since 1990, only three clinical studies of cannabinoids have been performed in acute pain, two in human volunteers $(11,12)$ and one on postoperative pain patients $(13)$.

\footnotetext{
${ }^{1}$ McGill University; ${ }^{2}$ Université de Montréal, Montreal, Quebec

Correspondence: Dr Mark Ware, E19.145 Montreal General Hospital, 1650 Cedar Avenue, Montreal, Quebec H3G 1A4.

Telephone 514-934-8222 ext 4386, fax 514-934-8096, e-mail mark.ware@muhc.mcgill.ca
} 
In 2000, Greenwald and Stitzer (11) reported a study of the antinociceptive properties of smoked cannabis in recreational drug users. The volunteers were healthy regular marijuana users. Of the 13 participants enrolled, only five male volunteers completed the entire study, which consisted of three test sessions, during which they smoked cigarettes containing 0\% (placebo) and 3.55\% delta-9-tetrahydrocannabinol (THC) (active). Cannabis smoking was divided in four bouts, during which participants inhaled nine puffs (zero, three, six or nine active puffs separated by $40 \mathrm{~min}$ each). Patients were also given naltrexone (an opioid antagonist) - $0 \mathrm{mg}, 50 \mathrm{mg}$ or $200 \mathrm{mg}$ orally $-1 \mathrm{~h}$ before the first smoking session, to assess whether endogenous opioids influence cannabis effects in humans. Test sessions consisted mainly of antinociceptive measures using finger withdrawal from radiant heat stimulation, a procedure in humans that is comparable with a tail-flick assay. Overall, cannabis produced significant dose-dependent antinociception that was not antagonized by naltrexone. The effect was weak and only significantly present at the highest dose. Despite these positive results, the authors argued that because all participants were relatively frequent cannabis users, a greater tolerance to the antinociceptive effects of cannabis smoking may have developed.

The analgesic effect of oral THC, morphine or their combination was reported in healthy subjects under experimental pain conditions (12). This was a randomized, placebo-controlled, double-blind, crossover study involving 12 healthy cannabis-naïve volunteers (six female and six male). Each subject orally received either $20 \mathrm{mg}$ THC (dronabinol), $30 \mathrm{mg}$ morphine, a mixture of $20 \mathrm{mg}$ THC and $30 \mathrm{mg}$ morphine, or placebo as a single dose. The between-session washout phases were at least seven days. Pain tests consisting of pressure pain tolerance, heat pain (thermode), ice-cold immersion, and single and repeated transcutaneous electrical stimulation were performed every hour up to $8 \mathrm{~h}$ post-drug administration. Furthermore, side effects and vital functions were monitored, as well as plasma concentrations of THC and its metabolites. In the heat test, neither morphine nor THC produced any analgesic effect. In the cold test, morphine alone and the combination morphine/THC were analgesic, but not THC alone, whereas in the pressure test, only morphine alone was analgesic. Finally, in the electrical stimulation test, morphine increased the pain detection threshold during single mode stimulation, while morphine alone and in combination with THC was analgesic during the repeated mode of stimulation. Drug administration was usually associated with mild side effects, with most patients feeling sleepy and confused after $\mathrm{THC}$ or $\mathrm{THC} /$ morphine administration. Interestingly, side effects of THC (in particular, euphoria, hallucinations and confusion) were lowered in the presence of morphine, and in the reverse, THC decreased nausea and vomiting associated with morphine when the two were given together. Taken together, these results illustrate that THC provides poor pain control in this battery of acute pain tests, which are characteristic of superficial pain (heat test and electrical stimulation) and more deep pain (pressure and cold test). However, opioids and non-steroidal anti-inflammatory drugs are known to provide no analgesia in the heat and cold tests, respectively. Furthermore, none of the experimental pain tests produced inflammation or tissue damage. Therefore, as the authors mentioned in their discussion, it is not possible to rule out the fact that THC would have an analgesic effect after induction of inflammation, or tissue or nerve damage. Finally, the plasma levels of THC in this study were very low; therefore, THC given with a better bioavailability could increase its analgesic effect and should be tested further.

A randomized clinical study (13) looking at postoperative pain showed a lack of efficacy of oral THC in women undergoing elective total abdominal hysterectomy. After surgery, all patients used a patient-controlled analgesia (PCA) device with morphine for $24 \mathrm{~h}$. After this time, the PCA was discontinued, and any patient requesting further analgesia was randomly assigned to receive either $5 \mathrm{mg}$ THC capsules or placebo. The primary outcome measure was the sum of the pain intensity differences over a $6 \mathrm{~h}$ period, while the second outcome measure was time to request for rescue analgesia in the form of oral codeine $(30 \mathrm{mg})$. Twenty patients were recruited in each group. No differences in mean sum of the pain intensity differences scores were found between the THC and placebo groups. The only side effect reported to be different between the groups was an increased awareness of surroundings in the THC group. The lack of efficacy of THC should be put into context of a study with a small number of patients using a small dose of THC as a single dose at day 2 after surgery. This study needs to be repeated using larger doses of THC (see below) or a combination of cannabinoids for a longer period of time before cannabinoids can be excluded from being effective in postoperative pain management.

\section{Chronic pain}

Ajulemic acid: Karst et al (14) conducted a small randomized controlled trial of 1',1'-dimethylheptyl-delta-8-THC-11-oic acid in 21 patients with chronic neuropathic pain. In this crossover study, $20 \mathrm{mg}$ of 1',1'-dimethylheptyl-delta-8-THC11 -oic acid twice daily for four days followed by $40 \mathrm{mg}$ twice daily for three days was associated with reduced pain measured $3 \mathrm{~h}$ after intake of the study drug compared with placebo. Eight hours after intake of the drug, the pain scale differences between groups were not significant. Adverse effects were transient dry mouth and tiredness, with no serious adverse event reported.

Cannabis extracts (sublingual): Berman et al (15) conducted a randomized controlled trial of two cannabis-based medicinal extracts for neuropathic pain resulting from brachial plexus avulsion. In this crossover trial, 48 patients received THC, a mixture of THC/cannabidiol (CBD) or placebo in a sublingual spray over a six-week period. Both THC and THC/CBD improved sleep quality, pain and quality of life.

Wade et al (16) conducted a series of double-blind, randomized, placebo-controlled, single-patient, crossover trials with two-week treatment periods in patients with intractable neurogenic symptoms. Patients had a range of disorders, including multiple sclerosis, spinal cord injury, brachial plexus avulsion and amputation. The patients were administered whole-plant extracts of THC, CBD, a combination of the two or a matched placebo by sublingual spray at doses determined by titration against symptom relief or unwanted effects within the range of $2.5 \mathrm{mg} / 24 \mathrm{~h}$ to $120 \mathrm{mg} / 24 \mathrm{~h}$. Pain relief from THC and CBD was superior to placebo, while impaired bladder control, muscle spasms and spasticity were improved in some patients. Three patients had transient hypotension and intoxication with rapid initial dosing of THC.

Notcutt et al (17) conducted a series of 'n-of-1' studies using a sublingual cannabis extract in 34 patients with chronic 
pain. Three cannabis-based medicine extracts (THC, CBD
and a 1:1 mixture of both) were given over a 12 -week period. After an initial open-label period, the cannabis-based medicine extracts were used in a randomized, double-blind, placebocontrolled, crossover trial. The extracts that contained THC proved most effective in symptom control. Regimens for the use of the sublingual spray emerged and a wide range of dosing requirements was observed. Side effects were common and were generally acceptable and similar to other psychoactive agents that are used for chronic pain.

As the present paper went to press, Health Canada granted conditional approval for the use of a buccal cannabis extract for chronic neuropathic pain associated with multiple sclerosis. Some of the data to support this have been cited above; other data are currently only available in abstract form or are awaiting publication. Prescribing information has been added to the recommendations section of this issue.

Cannabis extracts (oral): Zajicek et al (18) reported the results of a large-scale, multicentre, randomized, controlled trial of oral cannabinoids in the treatment of muscle spasticity associated with multiple sclerosis. Six hundred thirty participants were treated at 33 United Kingdom centres with oral cannabis extract $(n=211)$, THC $(n=206)$ or placebo $(n=213)$. The trial duration was 15 weeks. No treatment effect of cannabinoids was observed on the primary outcome of muscle spasticity measured by the Ashworth scale. There was evidence of a treatment effect on patient-reported spasticity and pain. Side effects were principally expected.

Svendsen et al (19) reported the results of a small, randomized controlled trial of oral THC (dronabinol) versus placebo in 24 patients with central pain associated with multiple sclerosis. Treatment duration was three weeks with up to $10 \mathrm{mg}$ of THC. Median pain intensity during the last week of treatment was significantly reduced compared with placebo (visual analogue scale 4.0 versus $5.0, \mathrm{P}=0.02$ ). Dizziness was the most frequent adverse event associated with THC, especially during the first week of treatment (19).

\section{Ongoing studies}

Two studies with different designs are underway in the same setting of postoperative pain and may shed light into the role of cannabinoids in postoperative pain. The first study, funded by the Medical Research Council (United Kingdom), is a multicentre study called CANPOP (Trial of Cannabis for Acute Post-Operative Pain). Preliminary results of a dose-finding study were presented in September 2003 at a European congress (European Federation of IASP Chapters) in Prague, Czech Republic. Patients undergoing major orthopedic surgery or hysterectomy were recruited and received a capsule from one of the four following groups: cannabis extracts containing mainly THC and CBD (Cannador, Society for Oncological and Immunological Research, Germany), a synthetic delta-9THC (dronabinol), a standard analgesic (acetaminophen) or a placebo. The primary objective of the study was to evaluate pain scores using a visual analogue scale for $6 \mathrm{~h}$ following the end of surgery. The dose-finding study showed that a dose of 10 mg of THC (Cannador) was effective (30 patients completed the study and 15 required rescue analgesia), whereas a dose of $5 \mathrm{mg}$ was not (the first 11 patients requested rescue analgesia, so the dose was stopped). At the $10 \mathrm{mg}$ dose, 29 patients were without nausea and one patient had moderate nausea for $2 \mathrm{~h}$. Based on these results, a full-scale study has begun using THC
$10 \mathrm{mg}$, and definitive results should be available in a few months.

Another study, funded by the Canadian Institute of Health Research, has recently started and is testing the role of a synthetic analogue of THC, nabilone, in postoperative pain, nausea and vomiting (Pierre Beaulieu, Université de Montréal Centre hospitalier de l'Université de Montréal, Québec). Patients undergoing major (orthopedic, gynecological, plastic or urological) surgery receive capsules of either $1 \mathrm{mg}$ or $2 \mathrm{mg}$ of nabilone, ketoprofen or placebo every $8 \mathrm{~h}$ for $24 \mathrm{~h}$ while simultaneously receiving morphine PCA. The primary objectives are total quantity of morphine used in $24 \mathrm{~h}$ and pain scores at rest and on movement. Finally, the incidence of nausea and vomiting, tolerance of the study medications and quality of sleep are also recorded. This prospective, randomized, doubleblind study will recruit a total of 160 patients.

For chronic pain, one pilot, randomized, placebo-controlled study of smoked cannabis is underway at the McGill University Health Centre, Montreal, Quebec. Thirty-two subjects are to be randomly assigned to receive four different cannabis preparations ranging in THC content from $0 \%$ to 9.5\%. Subjects will be allocated to receive all four preparations in a crossover design trial. In each cycle, they will use $25 \mathrm{mg}$ three times daily for five days, on an outpatient basis, with nine-day washout periods between cycles. The primary outcome is average pain intensity measured during the smoking phase. The study is expected to be completed in late 2005.

An additional study is underway in patients with HIV. associated neuropathic pain in San Francisco, California, USA. Preliminary findings of an open-label phase suggest that patients using smoked cannabis obtain some pain relief, but the data have not yet been published.

\section{DISCUSSION}

The present review was not conducted systematically and only refers to studies available through the MEDLINE database. Important trial results or other data that may exist elsewhere may have been missed. However, the publications cited in the present paper are not uniformly positive, and the possibility of publication bias, while always a concern with controversial drugs like cannabis, is assumed to be minimal because the therapeutic potential is of great interest to physicians and regulators, and the direction of any potential bias is not obviously apparent.

These reports suggest that there is some therapeutic potential for cannabinoids in acute and chronic pain, but that significant hurdles exist in achieving clinically relevant outcomes with minimal side effects. Much more work is needed to evaluate appropriate outcomes, to develop alternative means of administration and to develop dosing strategies. Further work is needed using new and existing cannabinoids to continue to explore the therapeutic potential of cannabinoids in chronic pain.

As with morphine and nonsteroidal anti-inflammatory drugs, cannabinoids may not be very effective in experimental acute pain. Their role in postoperative pain may be more attractive in the presence of tissue injury and inflammation. Cannabinoids may act as adjuvants to opioids to control pain, and also nausea and vomiting (20), which are not trivial symptoms in the postoperative period. From the current evidence, when given in combination, opioids and cannabinoids may reduce each other's side effects and produce an adequate combination in the treatment of acute pain. 


\section{COPYRIGHT PULSUS GROUP INC, " DO NOT COPY}

For chronic pain, the evidence is mounting that cannabinoids may constitute a new class of agents to add to the pharmaceutical toolbox in the management of chronic pain. The effects of cannabinoids on pain, including peripheral and central neuropathic pain states, and spasticity are measurable and meaningful to patients, and suggest that with appropriate prescribing and monitoring, additional benefit to some patients may be expected with cannabinoid therapy. Short-term side effects are well described, and often are the main drawbacks to patient compliance with therapy. More specific information is required on the long-term side effects of cannabinoid therapy, including drugdrug interactions, tolerance, cognitive impairment and risks of addiction, but these issues are not unique to cannabinoids and indeed point to the need for physicians to exercise diligence in following patients on psychoactive medications.

\section{SUMMARY}

The past two years have seen the publication of several important clinical trials that point to the potential role for cannabinoids in pain management, and ongoing work promises to further explore and define these effects. Cannabinoids

\section{REFERENCES}

1. Meng ID, Manning BH, Martin WJ, Fields HL. An analgesia circuit activated by cannabinoids. Nature 1998;395:381-3.

2. Ware MA, Doyle CR, Woods R, Lynch ME, Clark AJ. Cannabis use for chronic non-cancer pain: Results of a prospective survey. Pain 2003;102:211-6.

3. Holdcroft A, Smith M, Jacklin A, et al. Pain relief with oral cannabinoids in familial Mediterranean fever. Anaesthesia 1997;52:483-6.

4. Chatterjee A, Almahrezi A, Ware M, Fitzcharles MA. A dramatic response to inhaled cannabis in a woman with central thalamic pain and dystonia. J Pain Symptom Manage 2002;24:4-6.

5. Lynch ME, Clark AJ. Cannabis reduces opioid dose in the treatment of chronic non-cancer pain. J Pain Symptom Manage 2003;25:496-8.

6. Campbell FA, Tramer MR, Carroll D, Reynolds DJ, Moore RA, McQuay HJ. Are cannabinoids an effective and safe treatment option in the management of pain? A qualitative systematic review. BMJ 2001;323:13-6.

7. Iversen L. Cannabinoids in pain management. Few well controlled trials of cannabis exist for systemic review. BMJ 2001;323:1250.

8. Price MAP, Notcutt WG. Cannabis and cannabinoids in pain relief. In: Brown DT, ed. Cannabis: The Genus Cannabis, 1st edn. Amsterdam: Harwood Academic Publishers, 1998:223-46.

9. Hays H. Marijuana for the management of proximal mytonic myopathy. J Pain Symptom Manage 2001;21:267-9.

10. Ware MA, Gamsa A, Persson J, Fitzcharles MA. Cannabis for chronic pain: Case series and implications for clinicians. Pain Res Manage 2002;7:95-9.

11. Greenwald MK, Stitzer ML. Antinociceptive, subjective and behavioral effects of smoked marijuana in humans. Drug Alcohol Depend 2000;59:261-75.

12. Naef M, Curatolo M, Petersen-Felix S, Arendt-Nielsen L, are worthy of consideration for refractory pain conditions, but it is important to fully inform patients who may embark on this line of therapy of the side effect profile, and to monitor doses used and adverse events carefully. Not surprisingly, the short-term risks are easier to evaluate than the long-term ones. The former do not appear to be excessive. Definite long-term risks exist, particularly for respiratory ailments, cognitive functions and psychosis. While long-term use may be quite sound, it requires active attention to an evolving topic.

FINANCIAL SUPPORT: MW is supported by the fonds de la recherche en santé (FRSQ) (Boursier-clinicien junior 1) and holds grants from the Canadian Institutes of Health Research (CIHR). MW has received honoraria from Valeant and AstraZeneca, has sat on advisory boards for Bayer and Valeant, has acted as a consultant to Cannasat, and has conducted research with grants from GW Pharmaceuticals and Valeant. PB is supported by the FRSQ (Boursier-clinicien junior 1), the Research Center of the Centre hospitalier de l'Université de Montréal and holds a grant from the CIHR.

Zbinden A, Brenneisen R. The analgesic effect of oral delta-9tetrahydrocannabinol (THC), morphine, and a THC-morphine combination in healthy subjects under experimental pain conditions. Pain 2003;105:79-88.

13. Buggy DJ, Toogood L, Maric S, Sharpe P, Lambert DG, Rowbotham DJ. Lack of analgesic efficacy of oral delta-9. tetrahydrocannabinol in postoperative pain. Pain 2003;106:169-72.

14. Karst M, Salim K, Burstein S, Conrad I, Hoy L, Schneider U. Analgesic effect of the synthetic cannabinoid CT-3 on chronic neuropathic pain: A randomized controlled trial. JAMA 2003;290:1757-62.

15. Berman JS, Symonds C, Birch R. Efficacy of two cannabis based medicinal extracts for relief of central neuropathic pain from brachial plexus avulsion: Results of a randomised controlled trial. Pain 2004 Dec;112:299-306.

16. Wade DT, Robson P, House H, Makela P, Aram J. A preliminary controlled study to determine whether whole-plant cannabis extracts can improve intractable neurogenic symptoms. Clin Rehabil 2003;17:21-9.

17. Notcutt W, Price M, Miller R, et al. Initial experiences with medicinal extracts of cannabis for chronic pain: Results from 34 ' $\mathrm{N}$ of 1' studies. Anaesthesia 2004;59:440-52.

18. Zajicek J, Fox P, Sanders H, et al. Cannabinoids for treatment of spasticity and other symptoms related to multiple sclerosis (CAMS study): Multicentre randomised placebo-controlled trial. Lancet 2003;362:1517-26.

19. Svendsen KB, Jensen TS, Bach FW. Does the cannabinoid dronabinol reduce central pain in multiple sclerosis? Randomised double blind placebo controlled crossover trial. BMJ 2004;329:253.

20. Tramer MR, Carroll D, Campbell FA, Reynolds DJ, Moore RA, McQuay HJ. Cannabinoids for control of chemotherapy-induced nausea and vomiting: Quantitative systematic review. BMJ 2001;323:16-21. 


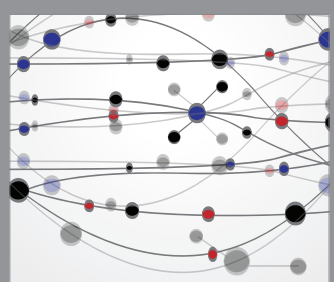

The Scientific World Journal
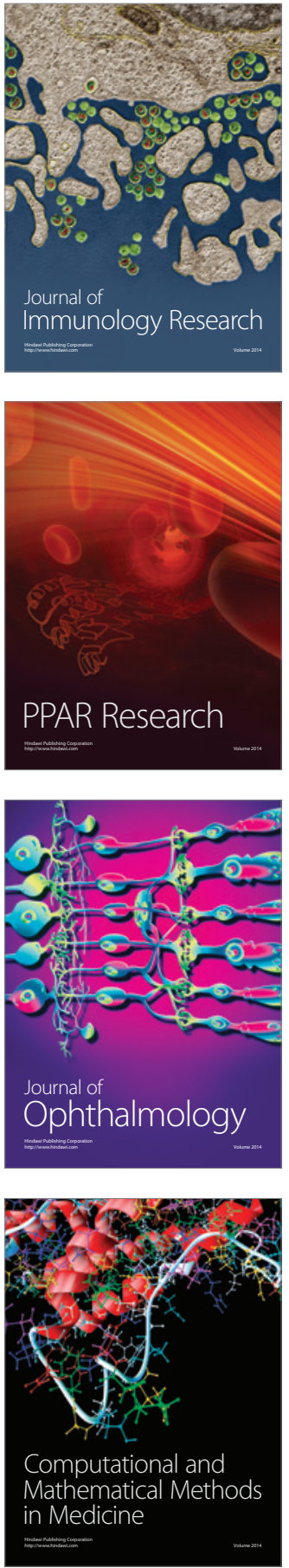



Gastroenterology Research and Practice

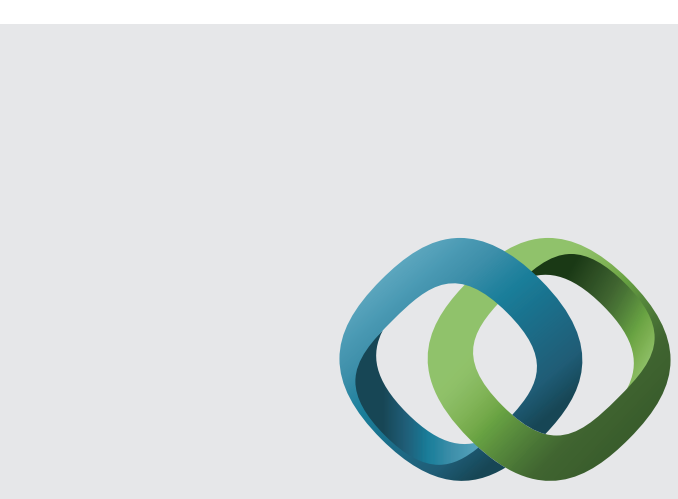

\section{Hindawi}

Submit your manuscripts at

http://www.hindawi.com

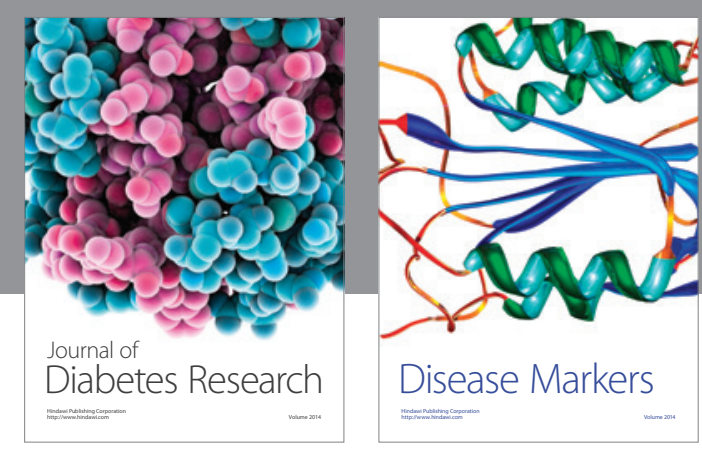

Disease Markers
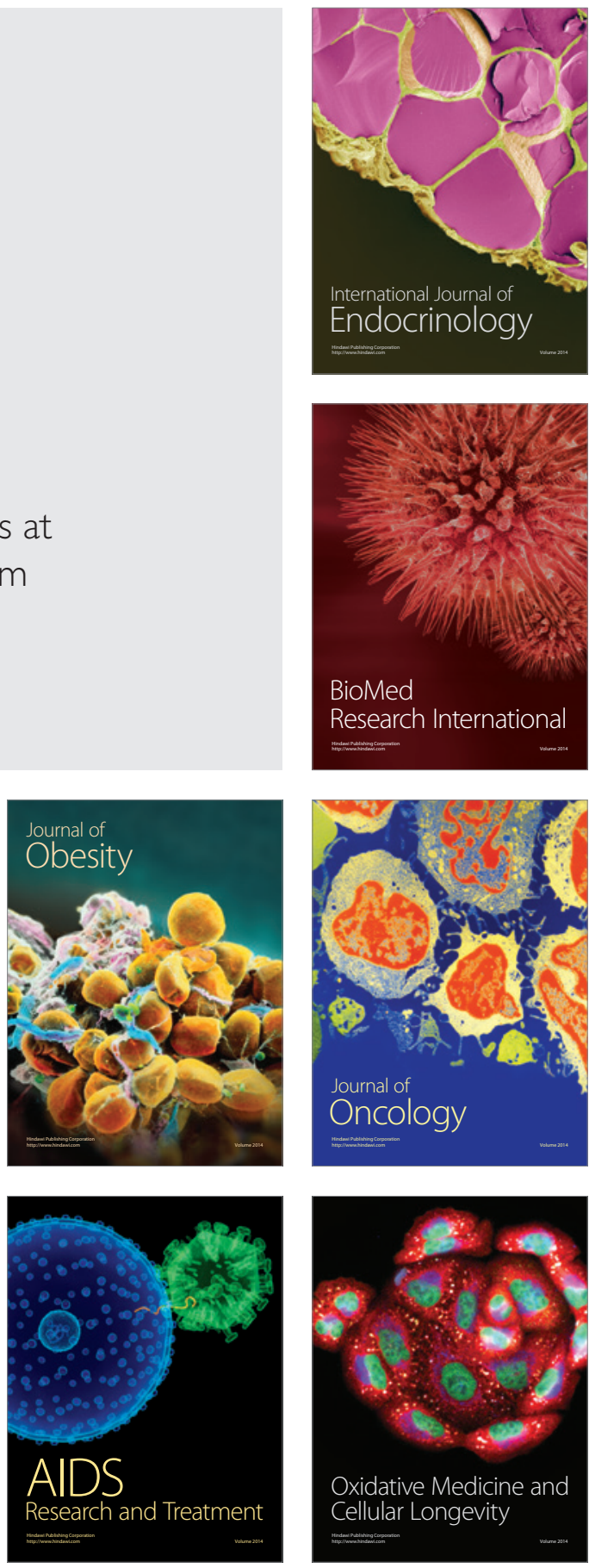